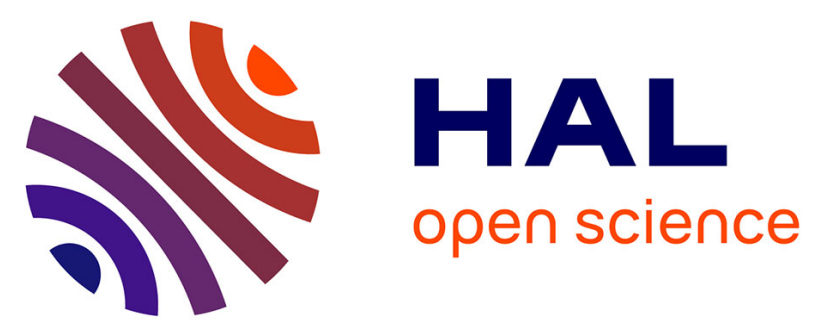

\title{
Identification des cancers mammaires triple-négatifs : analyse statistique de variables radiomiques issues des images TEP et de variables métabolomiques
}

Fanny Orlhac, Charles Bouveyron, Thierry Pourcher, Lun Jing, Jean-Marie Guigonis, Jacques Darcourt, Nicholas Ayache, Olivier Humbert

\section{To cite this version:}

Fanny Orlhac, Charles Bouveyron, Thierry Pourcher, Lun Jing, Jean-Marie Guigonis, et al.. Identification des cancers mammaires triple-négatifs: analyse statistique de variables radiomiques issues des images TEP et de variables métabolomiques. 2018 - 4èmes Journées Francophones de Médecine Nucléaire, May 2018, Lille, France. pp.169. hal-01736154

\author{
HAL Id: hal-01736154 \\ https://hal.science/hal-01736154
}

Submitted on 16 Mar 2018

HAL is a multi-disciplinary open access archive for the deposit and dissemination of scientific research documents, whether they are published or not. The documents may come from teaching and research institutions in France or abroad, or from public or private research centers.
L'archive ouverte pluridisciplinaire HAL, est destinée au dépôt et à la diffusion de documents scientifiques de niveau recherche, publiés ou non, émanant des établissements d'enseignement et de recherche français ou étrangers, des laboratoires publics ou privés. 
Identification des cancers mammaires triple-négatifs : analyse statistique de variables radiomiques issues des images TEP et de variables métabolomiques

Fanny Orlhac ${ }^{1}$, Charles Bouveyron ${ }^{1,2}$, Thierry Pourcher ${ }^{3}$, Lun Jing ${ }^{3}$, Jean-Marie Guigonis ${ }^{3}$, Jacques Darcourt $^{3,4}$, Nicholas Ayache ${ }^{1}$, Olivier Humbert ${ }^{3,4}$

1 : Epione, Inria Sophia-Antipolis, Valbonne, France

2 : Laboratoire JAD, UMR CNRS 7135/UCA, Nice, France

3 : Laboratoire TIRO, UMRE 4320 CEA/UCA, Nice, France

4 : Service de médecine nucléaire, Centre Antoine Lacassagne/UCA, Nice, France

Mots clés: Analyse de texture, Cancer du sein, Hétérogénéité tumorale, Traitement des images, Triple-négatif

Objectif : La caractérisation de l'hétérogénéité tumorale à partir des images TEP connaît un intérêt croissant. Or le lien entre les variables radiomiques (VR) et les caractéristiques biologiques des lésions est encore mal connu. Notre objectif est d'étudier la corrélation entre les VR et les variables métabolomiques (VM) dans le cancer du sein, et d'analyser leur capacité à prédire le sous-type immunohistochimique des lésions.

Matériels \& Méthodes : 26 patientes ayant un cancer mammaire ont bénéficié d'un examen TEP au 18F-FDG pré-traitement (Biograph TEP/TDM, Siemens). Pour chaque lésion tumorale primitive, 43 VR ont été calculées (logiciel LIFEx). A partir de la pièce opératoire, un spectromètre de masse a été utilisé pour mesurer l'expression de 1500 VM référencées dans la base Human metabolome. Les coefficients de corrélation de Spearman (R) entre chaque VR et chaque VM ont été analysés. Nous avons également étudié séparément la capacité des VR et VM pour identifier les lésions mammaires triple-négatives (TN), en comparant les performances de 5 méthodes statistiques avec celles de SUVmax et du volume métabolique. Cette procédure a été répétée 25 fois avec une sélection aléatoire de 16 patientes pour l'apprentissage et de 10 patientes pour la validation. Les performances de chaque méthode pour l'identification des lésions TN ont été mesurées en utilisant l'index de Youden (sensibilité+spécificité-1).

Résultats : Dans notre étude, 7 lésions sont TN. Le coefficient de corrélation moyen, en valeur absolue, entre les VR et VM est égal à 0,20 $\pm 0,14$ (intervalle : [0-0,81], 3\% avec $|R| \geq 0,50$ ). Pour l'identification des lésions TN à partir des index conventionnels en TEP, SUVmax conduit aux meilleures performances (Youden $=0,29 \pm 0,34$ ). En utilisant les différentes méthodes statistiques, l'index de Youden moyen varie entre 0,18 et 0,34 à partir des VM et entre -0,12 et 0,50 pour les VR. Les meilleures performances pour la distinction des lésions TN sont obtenues à partir des VR pour I'analyse discriminante en grande dimension (HDDA) avec Youden=0,50 $\pm 0,35$.

Conclusion : Dans le cancer du sein, nous avons montré une corrélation faible à modérée entre les VR issus des images TEP et les VM. Le recrutement de patientes supplémentaires est en cours pour confirmer ces résultats. L'analyse conjointe des VR et VM est à l'étude afin de bénéficier de la complémentarité des informations radiomiques et métabolomiques et ainsi d'améliorer les performances de classification. 